\title{
Electromagnetic Modelling of Raman Enhancement from Nanoscale Structures as a Means to Predict the Efficacy of SERS Substrates
}

\author{
Richard J. C. Brown, Jian Wang, and Martin J. T. Milton \\ Analytical Science Group, National Physical Laboratory, Teddington, Middlesex TW11 OLW, UK
}

Received 19 March 2007; Accepted 31 July 2007

Recommended by Christian Brosseau

The requirement to optimise the balance between signal enhancement and reproducibility in surface enhanced Raman spectroscopy (SERS) is stimulating the development of novel substrates for enhancing Raman signals. This paper describes the application of finite element electromagnetic modelling to predict the Raman enhancement produced from a variety of SERS substrates with differently sized, spaced, and shaped morphologies with nanometre dimensions. For the first time, a theoretical comparison between four major generic types of SERS substrate (including metal nanoparticles, structured surfaces, and sharp tips) has been performed and the results are presented and discussed. The results of the modelling are consistent with published experimental data from similar substrates.

Copyright (c) 2007 Richard J. C. Brown et al. This is an open access article distributed under the Creative Commons Attribution License, which permits unrestricted use, distribution, and reproduction in any medium, provided the original work is properly cited.

\section{INTRODUCTION}

Recent developments in techniques for the detection of single molecules have opened up new opportunities for research in analytical chemistry and the life sciences [1-3]. One technique that has a wide range of potential applications is surface enhanced Raman spectroscopy (SERS) [4-7]. SERS is a noninvasive technique that can detect and characterise both simple organic $[8,9]$, and complex biological molecules $[10,11]$ at ultralow, and even single molecular concentrations. The sensitivity of SERS is particularly important since there is a growing emphasis on trace-level detection in analytical research [12].

The basis of the SERS technique is the capacity of metallic substrates to support the propagation of surface plasmons with resonant frequencies in the visible region of the electromagnetic spectrum [13]. These surface plasmons act to enhance the native Raman signal by producing an increased electric field in the vicinity of the target molecule $[14,15]$. In addition to this "electromagnetic mechanism," Raman signals from target molecules are also enhanced by a more complex and less well-quantified "chemical" or "electronic" mechanism, although previous studies have shown that this is usually small in comparison to the electromag- netic effect [16]. Silver and gold are the most widely used materials for SERS substrates and are most commonly used in the form of spherical particles on the nanometre scale $[17,18]$. In addition to these metal nanoparticles, other substrate types (Figure 1), such as nanostructured surfaces, and the tip-surface geometries offered by scanning probe microscopies, are being used increasingly for SERS experimentation [19]. However, little work has been published that compares the fundamental characteristics of these different substrate types for enhancing Raman signals. Moreover, if candidate SERS substrates could be assessed, and optimised by modelling their electromagnetic characteristics, time and effort could be saved in laboratory preparation and experimental testing.

Much effort has been expended in modelling the mechanisms of electromagnetic scattering accurately $[20,21]$. It is a complex problem because the nature of the secondary waves generated by scattering are determined not only by the characteristics of the incident wave but also by the field generated by other scatterers nearby. Therefore, for most practical situations, such as those encountered in SERS, numerical methods are employed. As the field enhancement is strongly dependent on physical parameters, such as the surface morphology, the optical constants used to perform 


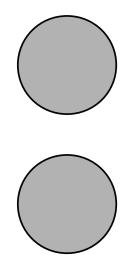

(a)

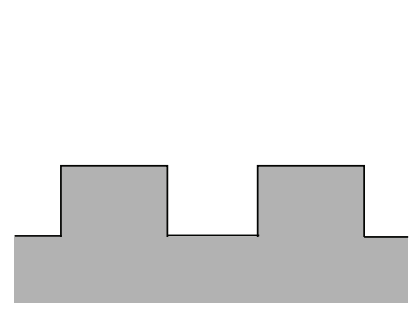

(c)

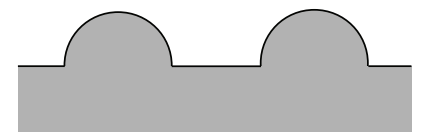

(b)

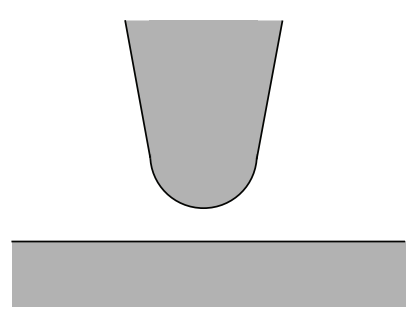

(d)
FIGURE 1: Diagrammatic representations of a section through the four generic types of SERS substrates modelled: (a) two nanoparticles, (b) structured surface with hemispherical features, (c) structured surface with cuboid features, and (d) tip surface.

the modelling, and the excitation conditions, detailed computational consideration is required to achieve robust models. Validation of these numerical results is challenging as direct experimental measurement of the field enhancement is usually not possible. However, we have previously shown that Raman enhancements from metal nanoparticles obtained experimentally agree with predictions from modelling to within one order of magnitude [16].

This paper presents the results of the predicted Raman enhancement, obtained using electromagnetic modelling, from four major generic types of SERS substrate. These generic substrates are shown diagrammatically in Figure 1.

The electric field enhancement between a pair of metal nanoparticles has received some study previously $[16,22]$, but the other substrates modelled here have received less attention. In this work, similar sizes and separations of features for each of the substrate types have been modelled so that the results may be compared. The results enable the relative enhancement from these commonly used substrates to be compared in detail for the first time.

\section{EXPERIMENTAL}

We have modelled the field distribution from four generic SERS metallic nanostructures in two dimensions. They can be considered as sections through a three-dimensional artefact at the point of maximum enhancement. For the substrates modelled here this will occur at the point of closest approach of the nanostructured features. For the two nanoparticles, this is along the line of their centres, and for a tipsurface substrate, this is in the plane passing through the centre of the point of tip. The substrates modelled are illustrated in detail in Figures 2, 3, 4, and 5. In all cases, silver substrates are considered and published optical constants for silver [23] that have been widely used for modelling studies have been

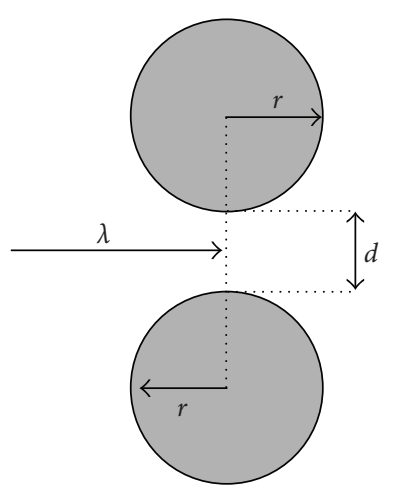

FIGURE 2: Section through two nanoparticles. The quantities varied during the modelling process were: $r$, the radius of the nanoparticles; $d$, the separation of the nanoparticles; and $\lambda$, the wavelength of the incident monochromatic radiation, which was incident normal to the line joining the centres of the nanoparticles.

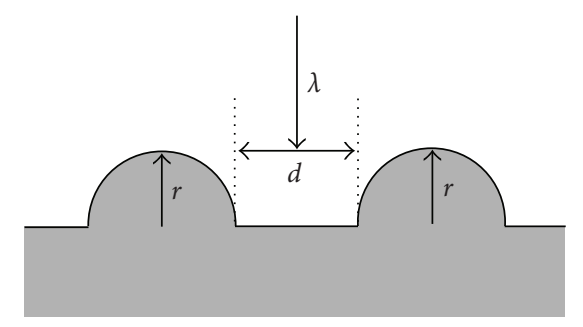

FIGURE 3: Section through the structured surface with hemispherical features. The quantities varied during the modelling process were: $r$, the radius of the metal hemispheres on the metal surface; $d$, the separation of the metal hemispheres; $\lambda$, the wavelength of the incident monochromatic radiation, which was incident normal to the metal surface.

employed. The polarisation of the incoming radiation (between 300 and $800 \mathrm{~nm}$ ) was in the incident plane to ensure excitation of surface plasmons. In the case of the structured surfaces, and the surface in the tip-surface substrate, the modelled structures are large enough that the maximum predicted enhancement is not influenced by the size of these boundaries.

The incident electromagnetic field in the complex-field representation can be written as follows:

$$
\begin{aligned}
\mathrm{E}(\mathbf{r}, t) & =\mathrm{E}_{0} \exp (i \mathrm{k} \cdot \mathbf{r}-i \omega t), \\
\mathrm{H}(\mathbf{r}, t) & =\mathrm{H}_{0} \exp (i \mathrm{k} \cdot \mathbf{r}-i \omega t),
\end{aligned}
$$

where $\mathrm{E}(\mathrm{r}, t)$ and $\mathrm{H}(\mathrm{r}, t)$ are the electric and magnetic field components, respectively, at location $\mathbf{r}$ and time $t ; \mathrm{E}_{0}$ and $\mathrm{H}_{0}$ are the electric and magnetic field amplitudes, respectively, $\mathrm{k}$ is the wavevector, and $\omega$ is the angular frequency of the wave. In the absence of nonlinear optical effects and when only the elastic scattering process is considered, the electromagnetic fields surrounding the scatterers must be selfconsistent, leading to the following conditions:

$$
\begin{aligned}
\mathrm{E}(\mathbf{r}, t) & =\mathrm{E}_{\text {in }}(\mathbf{r}, t)+\mathrm{E}_{\mathrm{sc}}(\mathbf{r}, t), \\
\mathrm{H}(\mathbf{r}, t) & =\mathrm{H}_{\text {in }}(\mathbf{r}, t)+\mathrm{H}_{\mathrm{sc}}(\mathbf{r}, t),
\end{aligned}
$$




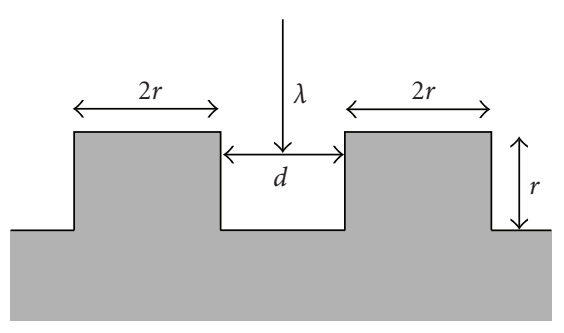

FIgURE 4: Section through the structured surface with cuboid features. The quantities varied during the modelling process were: $r$, the half-width, and the depth, of the metal cuboids on the metal surface; $d$, the separation of the metal cuboids; $\lambda$, the wavelength of the incident monochromatic radiation, which was incident normal to the metal surface. The corners of the structure have been rounded slightly, with radii of curvature of $1 \mathrm{~nm}$, to make the modelled situation more realistic.

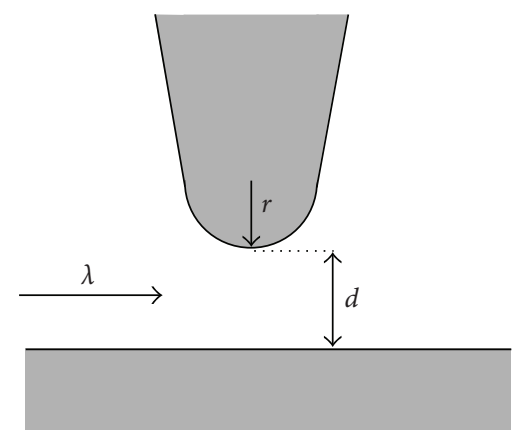

FIGURE 5: Section through the tip-surface substrate. The quantities varied during the modelling process were: $r$, the radius of the metal tip at its sharpest point; $d$, the separation of the metal tip from the surface; $\lambda$, the wavelength of the incident monochromatic radiation, which was incident parallel to the metal surface.

where the subscripts "in" and "sc" denote the incident and scattered waves, respectively. The electromagnetic waves must satisfy Maxwell's equations within the modelling domain, and adhere to the boundary equations at the interface between the media and the scatterer, leading to the following conditions:

$$
\begin{gathered}
\nabla \times(\nabla \times E)-\omega^{2} \varepsilon \mu E=0, \\
\nabla \times(\nabla \times H)-\omega^{2} \varepsilon \mu H=0, \\
\left(E_{2}(\mathbf{r})-E_{1}(\mathbf{r})\right) \times \mathbf{n}=0, \\
\left(H_{2}(\mathbf{r})-H_{1}(\mathbf{r})\right) \times \mathbf{n}=0,
\end{gathered}
$$

where the $\varepsilon$ and $\mu$ are the complex-valued permittivity and permeability and $\mathbf{r}$ is on the boundary of the scatterer, and $\mathbf{n}$ is a unit vector orthogonal to the boundary. The field distribution must be a solution of these equations. Finite element methods (FEM) using Comsol Multiphysics software [24] have been employed to provide numerical solutions to these equations for each substrate. Previous studies have also used FEM to model the field enhancement and spectral response in the vicinity of rough surfaces [25] and nanoparticles [26]. The FEM methodology requires the creation of a "radiation boundary condition" or "absorption boundary condition" to truncate the modelling scenario into a confined domain. The "perfect matched layers" (PML) boundaries method [27] has been used for this simulation. This matches the optical index at the interface of the media and attenuates the wave quickly within the artificial layer so that little or no electromagnetic radiation will be reflected back into the domain of scattering. Additionally, a low-reflection boundary condition is applied at the outer PML boundary in order to minimise residual reflection. In this way, the possibility of artefacts occurring in the modelling output is minimised.

The output of the modelling process is a two-dimensional map of the electric field intensity which can be used to calculate the Raman enhancement $G_{(r, \omega)}$ using [28]:

$$
G_{(r, \omega)}=\left|\frac{\widehat{E}_{\text {loc }}}{\widehat{E}_{\text {free }}}\left(\omega_{L}\right)\right|^{2}\left|\frac{\widehat{E}_{\text {loc }}}{\widehat{E}_{\text {free }}}(\omega)\right|^{2},
$$

where $\omega_{L}$ and $\omega$ are the frequencies of the incident and scattered light, respectively; $\widehat{E}_{\text {loc }}$ and $\widehat{E}_{\text {free }}$ are the absolute values of the local vector potentials (normalised to the intensities of the incident and scatted light) in the presence and absence, respectively, of the substrate. When the polarisation of the scattered light is the same as that of the incident light, the expected electromagnetic enhancement of the Raman signal may be expressed to a first approximation (for example Otto [28], Pendry [29], Moskovits [13], and Kneipp [30]) as

$$
G_{(r, \omega)}=\left|\frac{E_{(r, \omega)}}{E_{\text {inc }(\omega)}}\right|^{4},
$$

where $E_{(r, \omega)}$ is the total predicted electric field at position $r$, and $E_{\text {inc }(\omega)}$ is the electric field associated with the incoming electromagnetic radiation. The maximum value of the Raman enhancement $\left(G_{\max (r, \omega)}\right)$ can be calculated from any given situation. Three practical definitions of this maximum have been used in this work:

(a) the maximum value for $G_{(r, \omega)}$ obtained over the entire simulation area;

(b) the maximum value for $G_{(r, \omega)}$ obtained in a $5 \mathrm{~nm}^{2}$ area in the region of closest approach of the two metallic structures of interest;

(c) the average value for $G_{(r, \omega)}$ obtained in a $5 \mathrm{~nm}^{2}$ area in the region of closest approach of the two metallic structures of interest.

Figure 6 shows the maximum enhancement calculated according to these three definitions for two silver nanoparticles each with a radius of $25 \mathrm{~nm}$ and separated by $3 \mathrm{~nm}$. As can be seen, the three definitions lead to values of the Raman enhancement that are comparable to better than an order of magnitude at wavelengths longer than $350 \mathrm{~nm}$. At wavelengths shorter than $350 \mathrm{~nm}$, the maximum Raman enhancement is often predicted to be displaced from the region of closest approach of the two metallic structures of interest, therefore definition (a) produces higher enhancement factors. In order to avoid misinterpreting cases where the maximum Raman enhancement does not occur in the region of closest approach of the two metallic structures of interest, definition (a) was chosen as the method for calculating $G_{\max (r, \omega)}$ from the substrates presented here. 


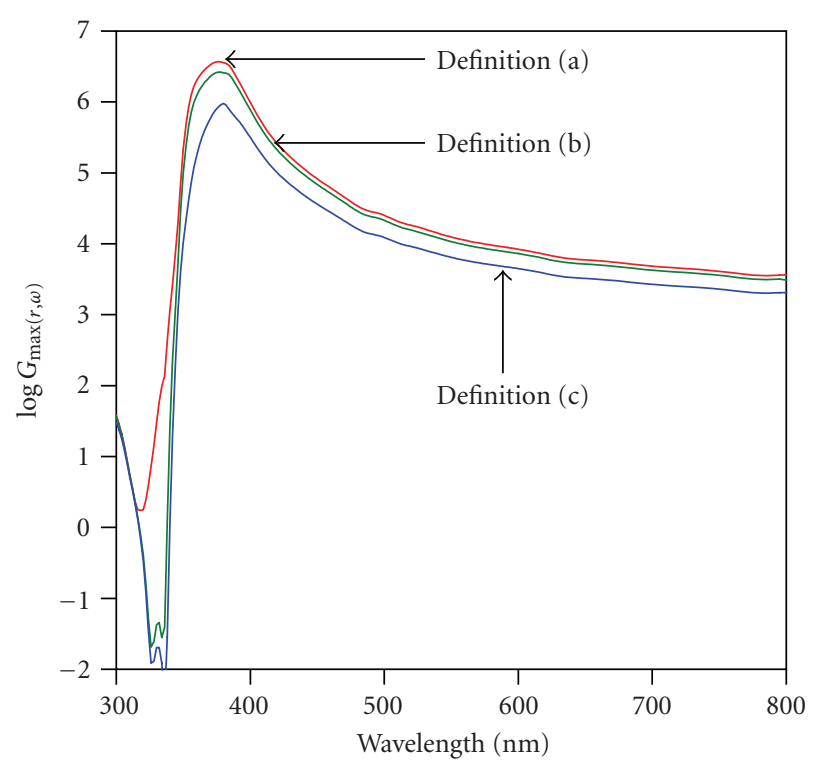

FIgURE 6: Wavelength dependence of the maximum Raman enhancement predicted using definition (a), (b), and (c) for two silver nanoparticles each with a radius of $25 \mathrm{~nm}$ separated by $3 \mathrm{~nm}$.

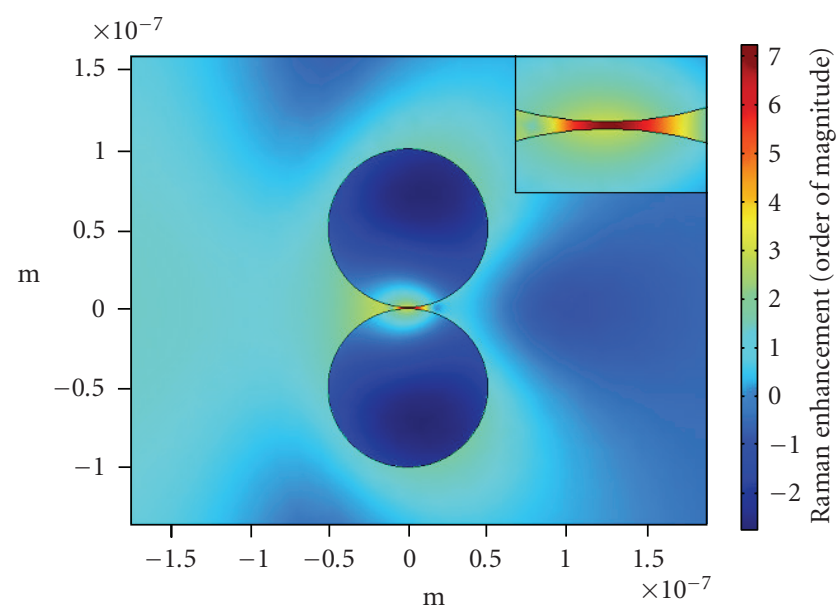

Figure 7: Modelled Raman enhancements around two silver nanoparticles; $r=50 \mathrm{~nm}, d=1 \mathrm{~nm} \lambda=514 \mathrm{~nm}$. The inset shows an enlarged portion around the area between the nanoparticles. The colours in red correspond to the areas of highest Raman enhancement and those in blue to the areas of lowest Raman enhancement.

Figure 7 shows an example of the distribution of predicted Raman enhancement around two silver nanoparticles.

Validation of the modelling procedure was carried out against published data; in particular, Garcia-Vidal's implementation of Maxwell's equations on adaptive meshes for the study of the interaction of light with metals surfaces [29] and with Xu et al.s study of colloid particle shape and size on the electromagnetic enhancement factor using classic electromagnetic theory [31]. In these cases, the methodology used here produced comparable enhancement factors to those predicted by Garcia-Vidal for structured metal surfaces for incident wavelengths of light between 310 and $620 \mathrm{~nm}$.
(Although these results were based on silver hemicylinders placed on a silver surface, a two-dimensional section through such a substrate, orthogonal to the cylinder axis, should be comparable to a similar section through hemispheres on a surface). The modelling used here also produces results that are comparable to Xu et al.'s predictions of enhancement factors for spherical particle substrates with separations of $1 \mathrm{~nm}$ and $5 \mathrm{~nm}$ (similar to the nanoparticles arrays being modelled in this study) to within an order of magnitude. This level of agreement with published data from two quite different studies confirms the applicability and flexibility of the modelling methodology used here for predicting Raman enhancement factors. Taking this data into consideration, the uncertainty in the predicted Raman enhancement data, within and between modelling scenarios, is approximately one order of magnitude. The uncertainty of the ratios of predicted enhancement factors within the same modelling scenario may be less than this.

\section{RESULTS AND DISCUSSION}

\subsection{Two nanoparticles}

The results of the modelling of the two nanoparticle substrate (Figure 2) is shown in Figure 8.

The principal trend in the modelled data is that predicted Raman enhancement increases as the separation of the features decreases. This relationship is strong- the difference in predicted maximum Raman enhancement from two nanoparticles $1 \mathrm{~nm}$ apart and $25 \mathrm{~nm}$ apart can be as much as six orders of magnitude. The maximum Raman enhancement predicted for these nanoparticles is of the same order of magnitude as the enhancement levels regularly observed experimentally by us and other groups $[16,32,33]$ approximately $10^{9}$. A comparison of the predicted results for different nanoparticle sizes in Figures 8(a), 8(b), and 8(c) shows that the maximum predicted enhancements are relatively similar. These trends are summarised in Figure 9.

Figure 9 illustrates the decrease in predicted maximum enhancement with increasing feature separation, and also shows that this relationship is relatively insensitive to feature size. It also shows that the wavelength at which the Raman maxima are predicted becomes shorter as the feature separation increases. Figure 10 shows that the average predicted

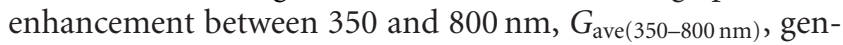
erally increases with feature size for any given feature separation.

\subsection{Structured surfaces}

Two nanostructured surfaces have been modelled; one with hemispherical features and the other with cuboid features. These are similar to substrates that can be produced by templating techniques (for the structured surfaces with hemispherical features) or e-beam lithography techniques (for the structured surfaces with cuboid features). The results of modelling the wavelength dependence of the maximum Raman enhancement for these surfaces are shown in Figures 11 and 12 , respectively. 


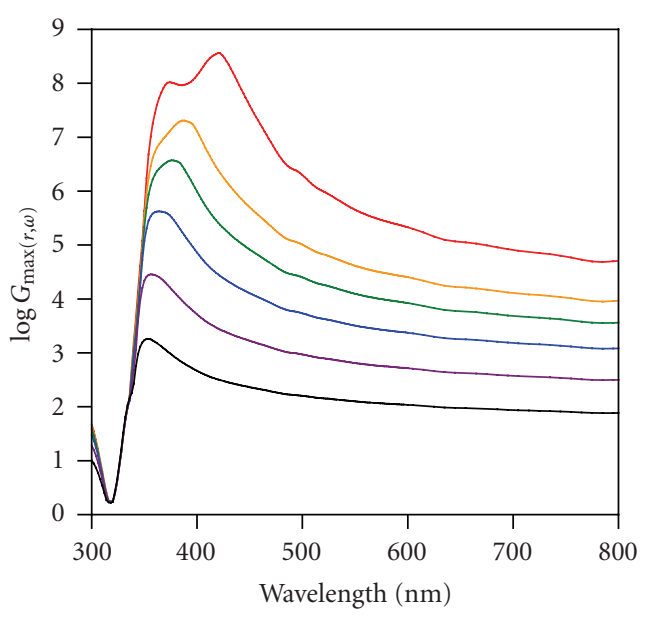

(a)

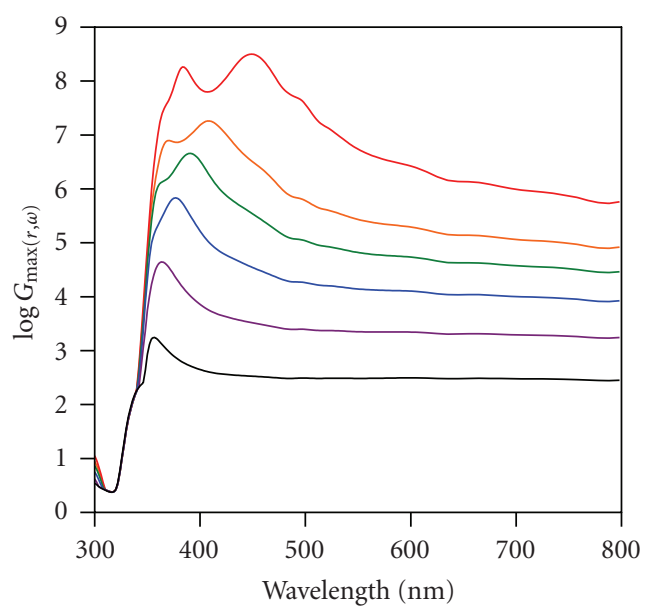

(b)

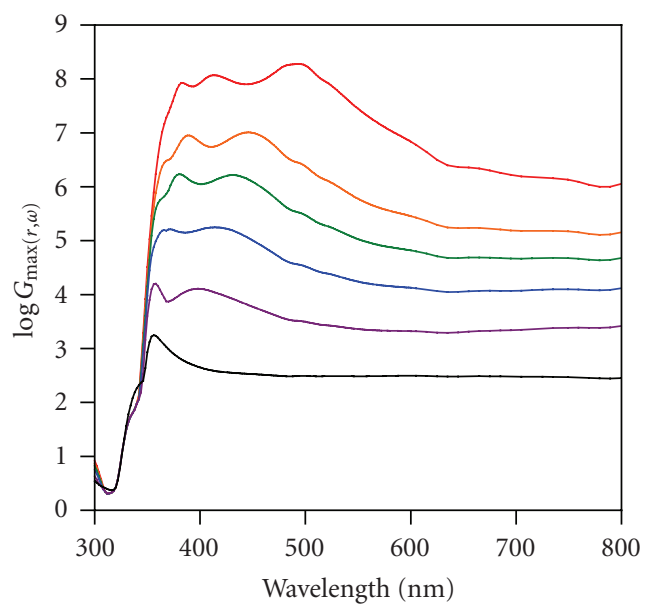

(c)

FIGURE 8: Modelled wavelength dependence of the maximum Raman enhancement from two silver nanoparticles, as shown in Figure 2, with radii $r$ of: (a), $25 \mathrm{~nm}$, (b), $50 \mathrm{~nm}$, and (c), $75 \mathrm{~nm}$. The separations of the two nanoparticles that have been modelled are: $1 \mathrm{~nm}$ (top curve), $2 \mathrm{~nm}, 3 \mathrm{~nm}, 5 \mathrm{~nm}, 10 \mathrm{~nm}$, and $25 \mathrm{~nm}$ (bottom curve).

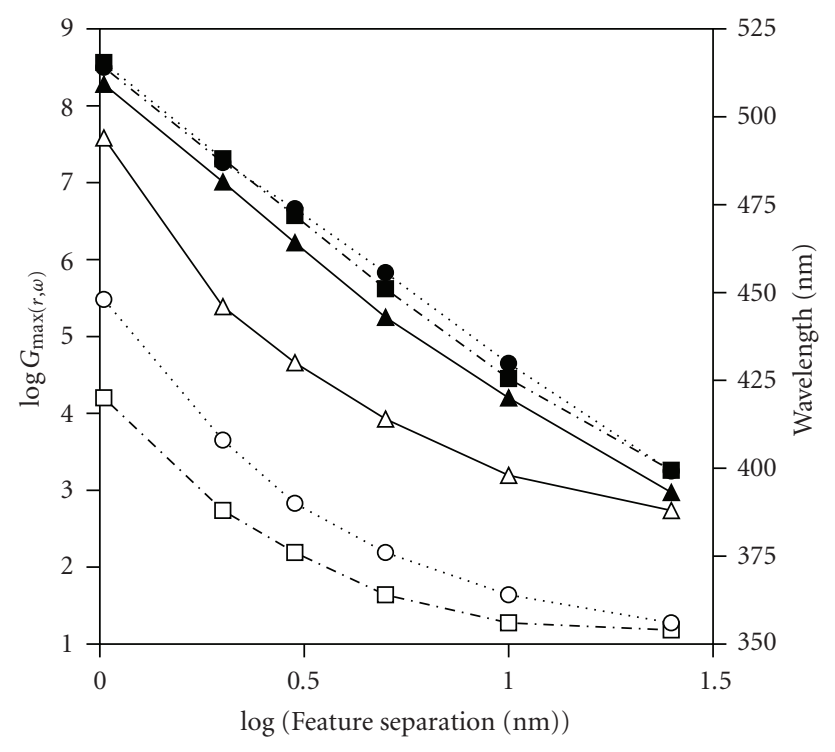

FIGURE 9: Relationship between the maximum predicted Raman enhancement and the separation of the two silver nanoparticles with radii of: $25 \mathrm{~nm}(\boldsymbol{\square}), 50 \mathrm{~nm}(\bullet)$, and $75 \mathrm{~nm}(\boldsymbol{\Delta})$. Additionally the dependence of the wavelength at which the maximum Raman enhancement is predicted to occur on the separation of the two silver nanoparticles with radii of: $25 \mathrm{~nm}(\square), 50 \mathrm{~nm}(\circ)$, and $75 \mathrm{~nm}(\triangle)$.

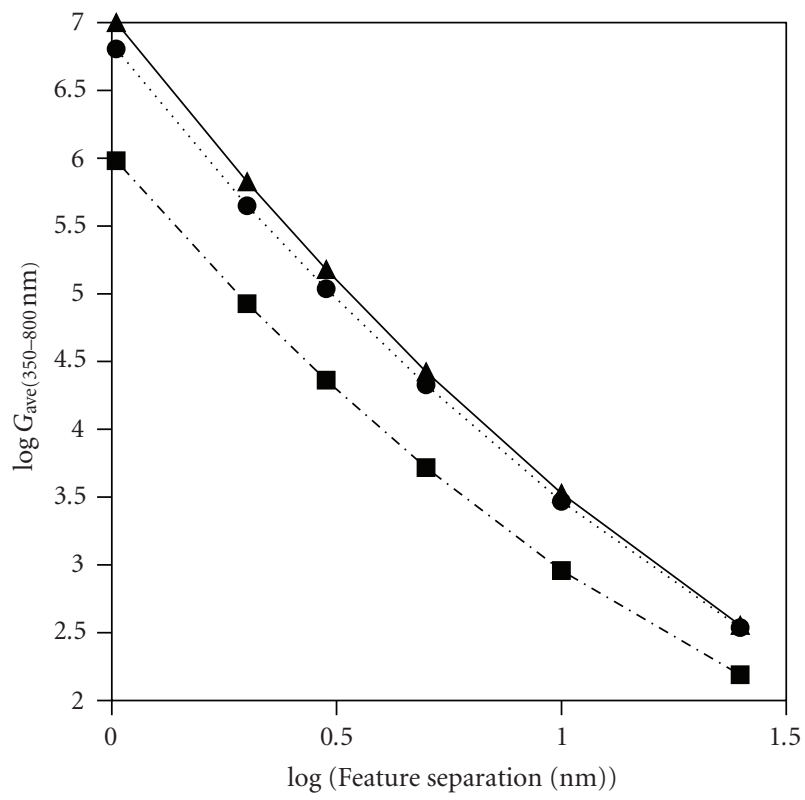

FIGURE 10: Relationship between the average predicted Raman enhancement between 350 and $800 \mathrm{~nm}$ and the separation of the two silver nanoparticles with radii of: $25 \mathrm{~nm}(\boldsymbol{\square}), 50 \mathrm{~nm}(\bullet)$, and $75 \mathrm{~nm}$ $(\boldsymbol{\Delta})$.

The results from the modelling of the surface with hemispherical features show several differences from the results for the two nanoparticles (Figure 8). In general, the enhancements observed are higher than those for the two nanoparticles at shorter wavelengths, but drop off more quickly as the wavelength increases and are generally lower than those 


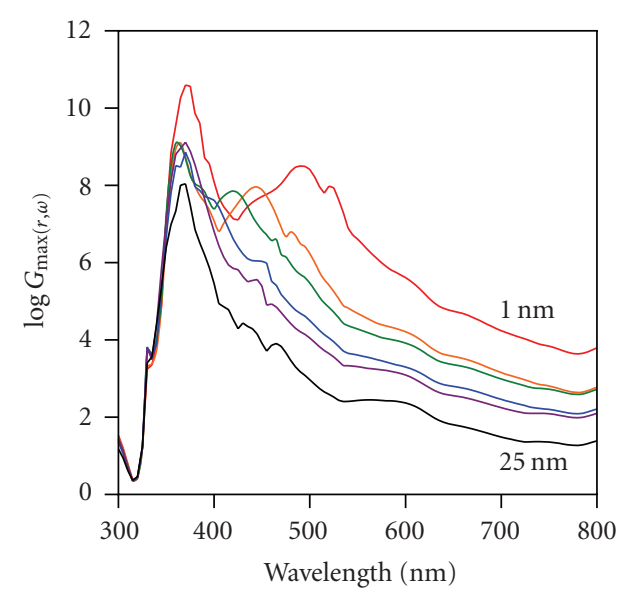

(a)

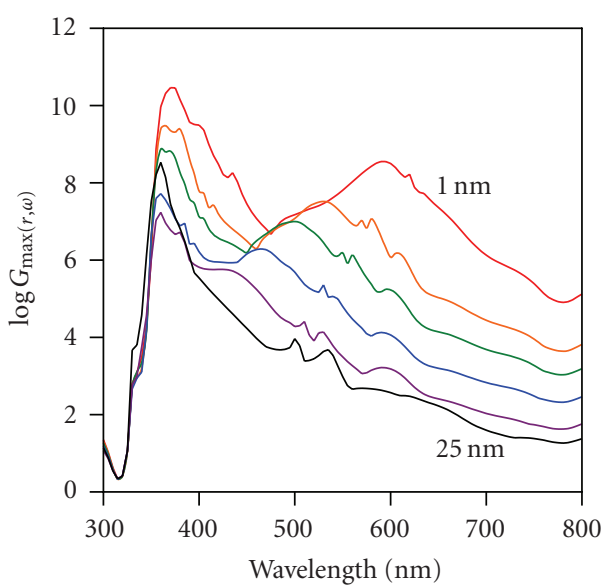

(b)

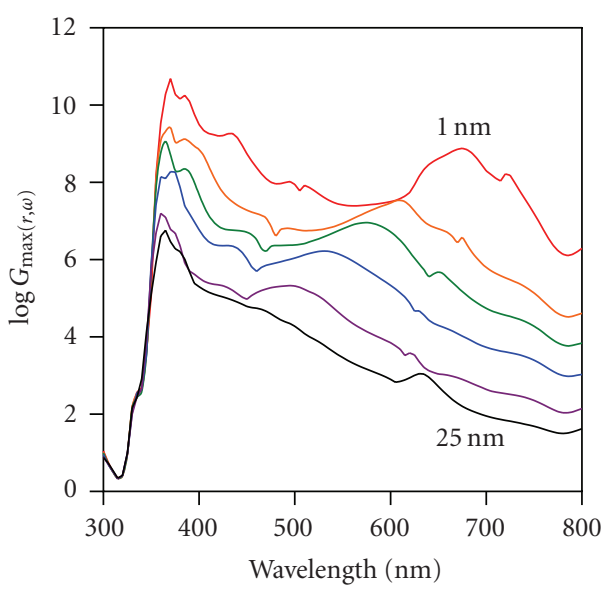

(c)

FIgURE 11: Modelled wavelength dependence of the maximum Raman enhancement from a structured silver surface with silver hemispherical features, as shown in Figure 3, with radii $r$ of: (a), $25 \mathrm{~nm}$, (b), $50 \mathrm{~nm}$, and (c), $75 \mathrm{~nm}$; on a flat silver surface. In each case, the separations of the hemispherical features are: $1 \mathrm{~nm}$ (top curve at long wavelengths), $2 \mathrm{~nm}, 3 \mathrm{~nm}, 5 \mathrm{~nm}, 10 \mathrm{~nm}$, and $25 \mathrm{~nm}$ (bottom curve at long wavelengths).

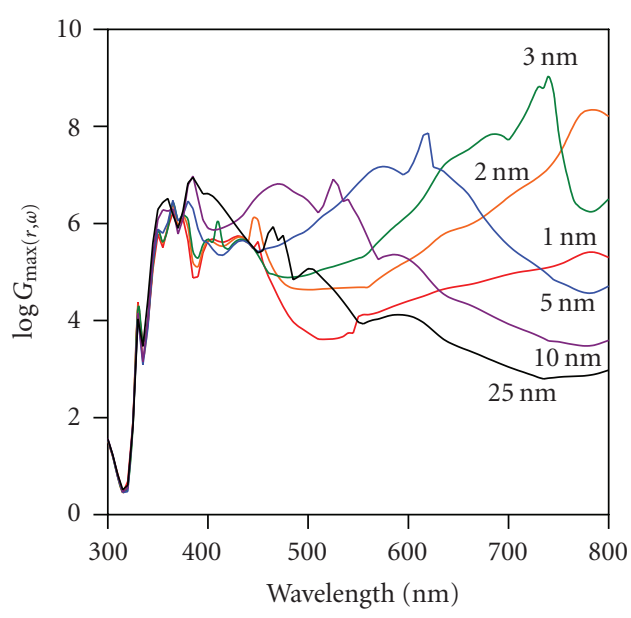

(a)

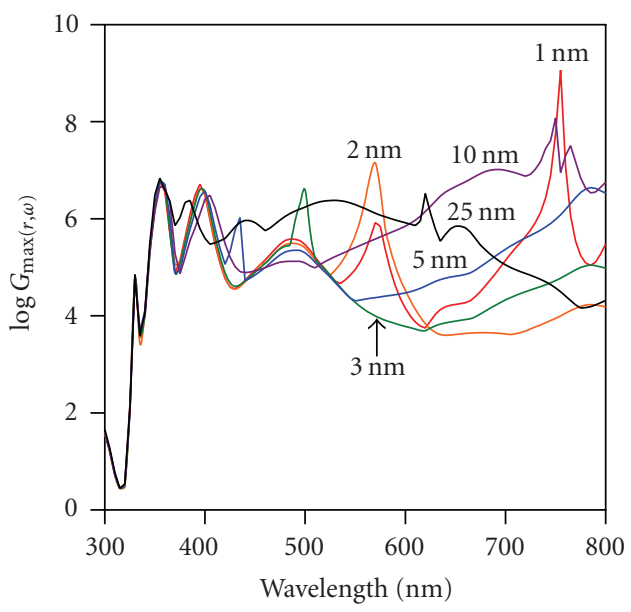

(b)

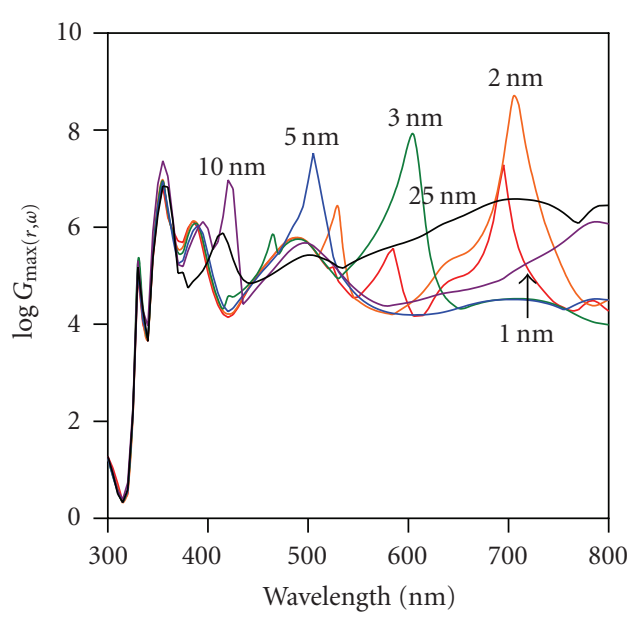

(c)

FIGURE 12: Modelled wavelength dependence of the maximum Raman enhancement from a structured silver surface with of silver cuboid features, as shown in Figure 4, with radii $r$ of : (a), $25 \mathrm{~nm}$, (b), $50 \mathrm{~nm}$, and (c), $75 \mathrm{~nm}$; on a flat silver surface. In each case the separations of the cuboid features are: $1 \mathrm{~nm}, 2 \mathrm{~nm}, 3 \mathrm{~nm}, 5 \mathrm{~nm}$, $10 \mathrm{~nm}$, and $25 \mathrm{~nm}$. 


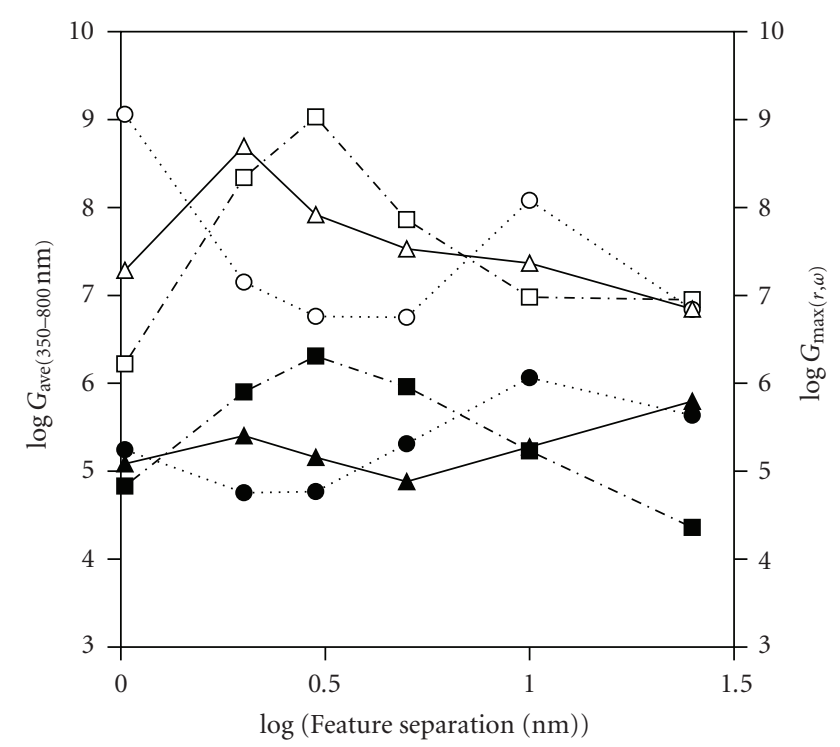

FIgURE 13: Relationship between the average predicted Raman enhancement (between 350 and $800 \mathrm{~nm}$ ) and the separation of the cuboid features on the structured surface with half-width $r$ of: $25 \mathrm{~nm}(\boldsymbol{\square}), 50 \mathrm{~nm}(\bullet)$, and $75 \mathrm{~nm}(\boldsymbol{\Delta})$; and between the average maximum predicted Raman enhancement the separation of the cuboid features on the structured surface with half-width $r$ of: $25 \mathrm{~nm}(\square) ; 50 \mathrm{~nm}(\circ)$; and $75 \mathrm{~nm}(\triangle)$.

observed for the two nanoparticles at longer wavelengths. The predicted enhancements from the structured surfaces with hemispherical features show a more complex dependence on wavelength than that observed for the two nanoparticles. The maximum enhancements predicted are less sensitive to feature separation than that for the two nanoparticles. Average enhancements from 350 to $800 \mathrm{~nm}$ drop off rapidly with increasing feature separation, but are generally an order of magnitude higher than for the two nanoparticles. The enhancements predicted correspond well with those observed in experimental studies for metal film over nanosphere (MFON) [34] and nanoembossed substrates [35].

The enhancement characteristics of the structured surface with cuboid features show a complex dependence on separation and wavelength (Figure 12). Most strikingly the predicted enhancements do not decrease monotonically with increasing wavelength, and with increasing feature separations. In many cases, the highest predicted enhancements are at longer wavelengths than for the other substrates, and for structures that do not have the smallest feature separations. The modelling predicts a slight decrease in the predicted enhancement with increasing feature separation, but very little change in average enhancement levels. Moreover, the enhancement levels predicted are not inconsistent with those observed from similar surfaces in experimental studies $[36,37]$ and from other modelling investigations [38]. These observations are illustrated by the relationship between the predicted enhancements and feature separation shown in Figure 13.

\subsection{Tip surface}

The results of modelling the wavelength dependence of the maximum Raman enhancement for the different tip-surface separations (Figure 5) are shown in Figure 14.

The enhancements observed from the tip-surface substrate show similar levels and wavelength trends to those predicted for the two nanoparticles. However, as the incident wavelength increases, the predicted enhancement shows a more complex wavelength dependence. Rather than a monotonic decrease in predicted enhancement from short to long wavelengths, at longer wavelengths the maximum enhancement actually shows an increase (particularly for the larger tip-surface separations). As the size of the tip radius increases there is a small increase in predicted overall enhancement levels. The tip-surface substrate shows very similar average and maximum predicted enhancements across the wavelengths as seen with the two nanoparticles. The predicted enhancements for the tip-surface substrate agree well with experimental data in the literature [39].

\subsection{Comparison of the four generic substrate types}

The four generic substrate types are compared in Figure 15 for features of $r=50 \mathrm{~nm}$ and separations of $1 \mathrm{~nm}$. The trends observed for $75 \mathrm{~nm}$ feature sizes best illustrate the relationships between the four substrates, however the trends for feature sizes of $50 \mathrm{~nm}$ and $25 \mathrm{~nm}$ are similar.

As can been seen in Figure 15(a), enhancements decrease as feature separations increase for all four substrates. In general, the structured surfaces produce larger enhancements across all feature separations, whilst the predicted enhancement from the tip surface and the two nanoparticles decreases sharply with increasing feature separation. The structured surfaces remain very efficient Raman enhancers, even at larger feature separations. Figure 15(b) shows that the average Raman enhancement decreases with increasing feature separation for all substrates except the structured surface with cuboid features. For this substrate, the average enhancement is relatively low but remains constant even up to feature separations of $25 \mathrm{~nm}$.

Figure 16 shows how the wavelength at which the maximum Raman enhancement is predicted varies with feature separation for the four substrates. In general, the wavelength of maximum predicted enhancement decreases as the feature separation increases, with all four substrates displaying a wavelength of maximum enhancement between 375 and $400 \mathrm{~nm}$ at a feature separation of $25 \mathrm{~nm}$. However, the rate at which this decrease occurs increases in the following order: structured surface with hemispherical features $<$ tip surface $<$ two nanoparticles $\ll$ structured surface with cuboid features.

\section{CONCLUSIONS}

The relative SERS performance of four generic substrates types that are commonly used in SERS have been modelled and their predicted Raman enhancements compared. Whilst the substrates modelled here only represent simplified 


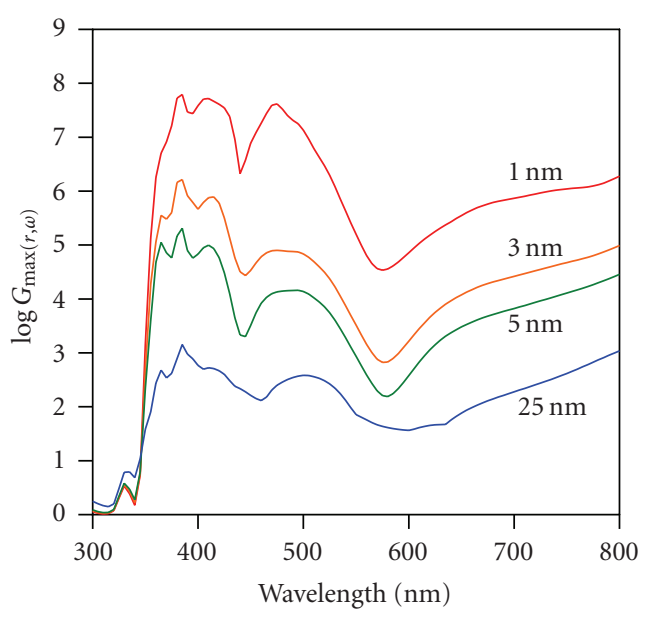

(a)

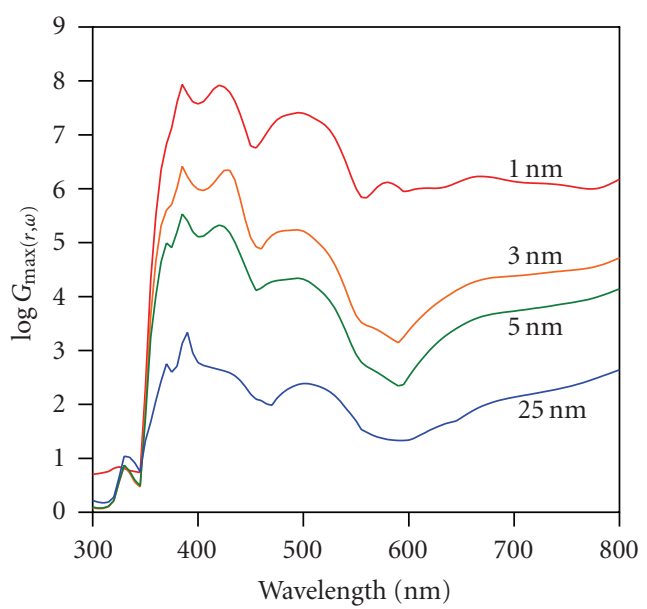

(b)

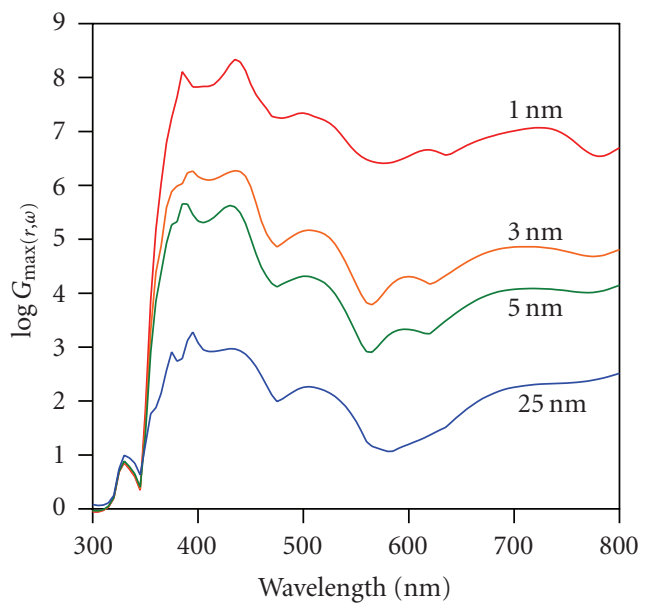

(c)

FIGURE 14: Modelled wavelength dependence of the maximum Raman enhancement predicted from silver tips and a silver surface, as shown in Figure 5, of end radii $r$ of: (a), $25 \mathrm{~nm}$, (b), $50 \mathrm{~nm}$, and (c), $75 \mathrm{~nm}$; in close proximity to a silver surface. In each case the separations of the tip and the surface that have been modelled are: $1 \mathrm{~nm}$, $3 \mathrm{~nm}, 5 \mathrm{~nm}$, and $25 \mathrm{~nm}$.

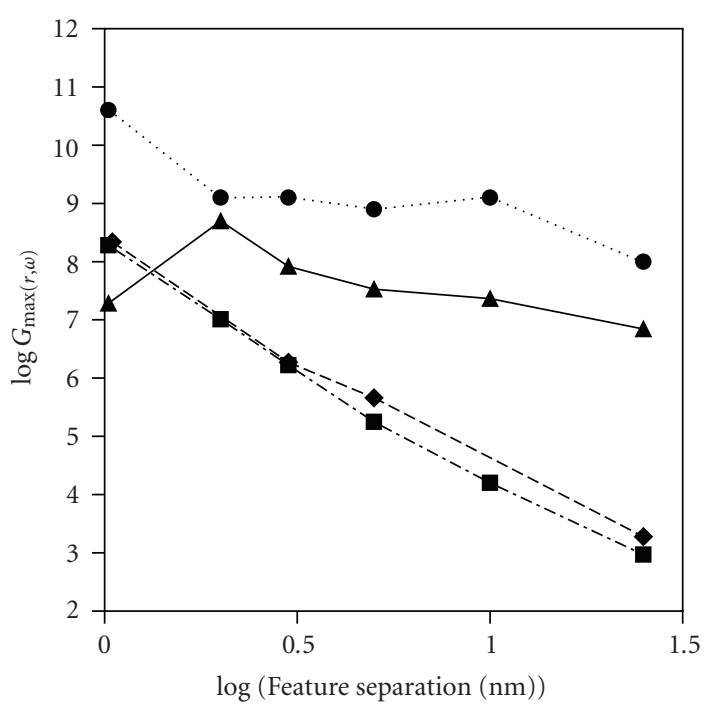

(a)

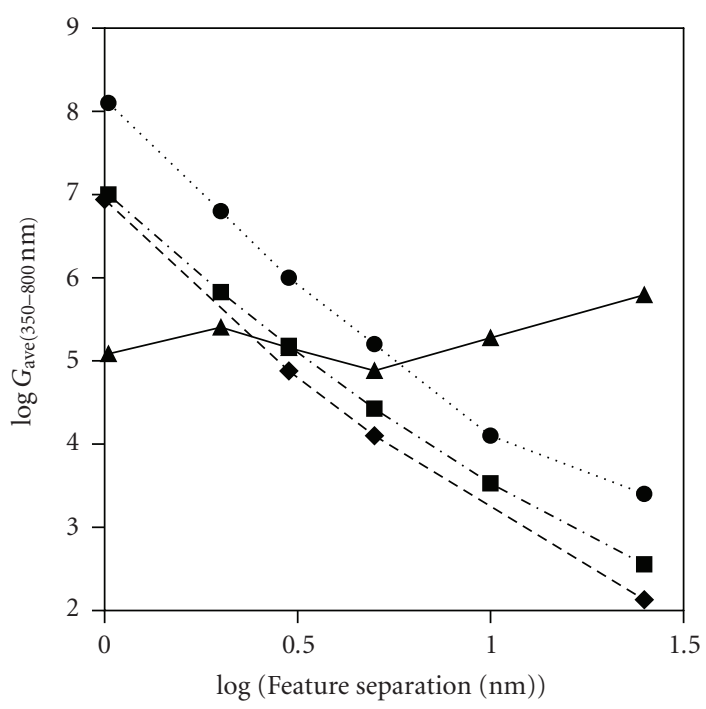

(b)

FIGURE 15: Maximum (Figure 15(a)) and average (between 350 and $800 \mathrm{~nm}$ ) (Figure 15(b)) predicted Raman enhancements, against feature separation for: two nanoparticles ( $\mathbf{\square})$; structured surface with hemispherical features $(\bullet)$; structured surface with cuboid features $(\boldsymbol{\Delta})$; and tip-surface $(\checkmark)$, for features of $r=75 \mathrm{~nm}$.

approximations of real experimental situations, the levels of enhancement predicted agree, in most cases, within an order of magnitude of what has been observed experimentally in the literature.

The study has shown that the predicted Raman enhancement is very sensitive to substrate feature separation over the range modelled ( $1 \mathrm{~nm}$ and $25 \mathrm{~nm}$ ). In addition, average enhancements across the wavelength range modelled (300$800 \mathrm{~nm}$ ) decrease with increasing feature separation, with the exception of the structured surface with cuboid features, which shows little change in average enhancement levels. Comparison of the four different generic substrate types 


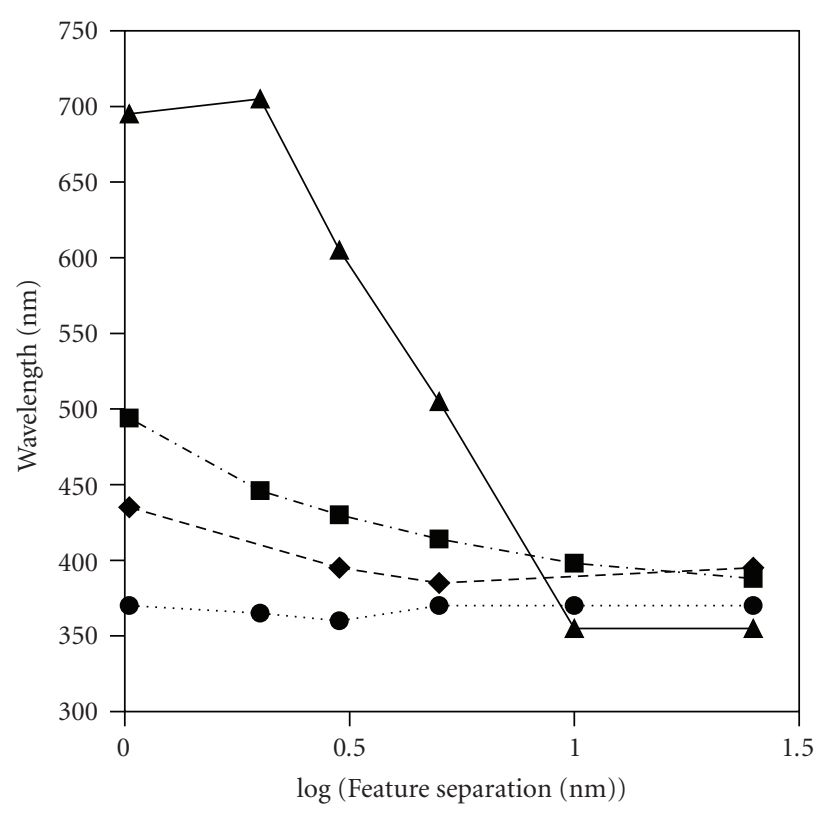

FIGURE 16: Relationship between the wavelength at which the predicted Raman enhancements is at a maximum and feature separation for: two nanoparticles ( $\mathbf{\square})$; structured surface with hemispherical features $(\bullet)$; structured surface with cuboid features $(\boldsymbol{\Delta})$; and tip-surface $(\diamond)$, for features of $r=75 \mathrm{~nm}$.

has revealed that structured surfaces produce larger maximum enhancements than the two nanoparticle or tip-surface substrates. Moreover, the structured surfaces show the most complicated relationships between predicted enhancement and wavelength. The behaviour of two nanoparticle and tipsurface substrates is similar and they produce comparable enhancement levels.

The results presented here predict that a structured surface with hemispherical features produces the largest maximum enhancements at all feature separations and the largest average enhancements at small feature separation. However, the structured surfaces with cuboid features show the most consistent maximum and average enhancements over all feature separations, and the largest average enhancements at large feature separations. An especially important finding of this work is that the average predicted enhancement from a structured surface with cuboid features over the wavelengths modelled shows very little sensitivity to feature size or separation. This suggests that it may not be necessary to manufacture these as precisely as was previously thought, indicating that they could perhaps be prepared to poorer tolerances, with little impact on their efficacy as substrates.

Experimentally, fabrication of structured surface is more time consuming and expensive than the preparation of metal nanoparticles. Tip-surface substrates are even more difficult to implement, but may provide the only way of performing robust SERS mapping of a surface, because the enhancement of the Raman signal is localised at the tip position. Therefore, a balance must be found between performance, reproducibility, and cost effectiveness of these substrates. It is clear that design and modelling of these surfaces prior to manufacture can save a large amount of experimental effort in this optimisation process. This work has shown that using the techniques presented here, robust and accurate model of SERS substrates is possible that can contribute to improving the efficiency of the research and development process in SERS.

\section{REFERENCES}

[1] R. Petry, M. Schmitt, and J. Popp, "Raman spectroscopy-a prospective tool in the life sciences," ChemPhysChem, vol. 4, no. 1, pp. 14-30, 2003.

[2] H. G. M. Edwards, "Probing history with Raman spectroscopy," The Analyst, vol. 129, no. 10, pp. 870-879, 2004.

[3] A. J. de Mello, "Seeing single molecules," Lab on a Chip, vol. 3, no. 2, pp. $29 \mathrm{~N}-34 \mathrm{~N}, 2003$.

[4] A. M. Michaels, M. Nirmal, and L. E. Brus, "Surface enhanced Raman spectroscopy of individual rhodamine $6 \mathrm{G}$ molecules on large Ag nanocrystals," Journal of the American Chemical Society, vol. 121, no. 43, pp. 9932-9939, 1999.

[5] S. R. Emory and S. Nie, "Near-field surface-enhanced Raman spectroscopy on single silver nanoparticles," Analytical Chemistry, vol. 69, no. 14, pp. 2631-2635, 1997.

[6] D. Zeisel, V. Deckert, R. Zenobi, and T. Vo-Dinh, "Nearfield surface-enhanced Raman spectroscopy of dye molecules adsorbed on silver island films," Chemical Physics Letters, vol. 283, no. 5-6, pp. 381-385, 1998.

[7] P. Etchegoin, H. Liem, R. C. Maher, et al., "A novel amplification mechanism for surface enhanced Raman scattering," Chemical Physics Letters, vol. 366, no. 1-2, pp. 115-121, 2002.

[8] C. Rodger, V. Rutherford, P. C. White, and W. E. Smith, “Towards quantitative surface enhanced resonance Raman scattering (SERRS): a study of aggregation and concentration for two rhodamine dyes," Journal of Raman Spectroscopy, vol. 29, no. 7, pp. 601-606, 1998.

[9] K. Kneipp, E. Roth, C. Engert, and W. Kiefer, "Near-infrared excited surface-enhanced Raman spectroscopy of rhodamine 6G on colloidal silver," Chemical Physics Letters, vol. 207, no. 4-6, pp. 450-454, 1993.

[10] P. Etchegoin, H. Liem, R. C. Maher, et al., "Observation of dynamic oxygen release in hemoglobin using surface enhanced Raman scattering," Chemical Physics Letters, vol. 367, no. 1-2, pp. 223-229, 2003.

[11] P. Etchegoin, R. C. Maher, L. F. Cohen, et al., "New limits in ultrasensitive trace detection by surface enhanced Raman scattering (SERS)," Chemical Physics Letters, vol. 375, no. 1-2, pp. 84-90, 2003.

[12] R. J. C. Brown and M. J. T. Milton, "Analytical techniques for trace element analysis: an overview," Trends in Analytical Chemistry, vol. 24, no. 3, pp. 266-274, 2005.

[13] M. Moskovits, "Surface-enhanced spectroscopy," Reviews of Modern Physics, vol. 57, no. 3, pp. 783-826, 1985.

[14] W. E. Doering and S. Nie, "Single-molecule and singlenanoparticle SERS: examining the roles of surface active sites and chemical enhancement," Journal of Physical Chemistry B, vol. 106, no. 2, pp. 311-317, 2002.

[15] C. H. Munro, W. E. Smith, M. Garner, J. Clarkson, and P. C. White, "Characterization of the surface of a citrate-reduced colloid optimized for use as a substrate for surface-enhanced resonance raman scattering," Langmuir, vol. 11, no. 10, pp. 3712-3720, 1995. 
[16] R. J. C. Brown, J. Wang, R. Tantra, R. E. Yardley, and M. J. T. Milton, "Electromagnetic modelling of Raman enhancement from nanoscale substrates: a route to estimation of the magnitude of the chemical enhancement mechanism in SERS," Faraday Discussions, vol. 132, pp. 201-213, 2006.

[17] R. C. Maher, L. F. Cohen, and P. Etchegoin, "Single molecule photo-bleaching observed by surface enhanced resonant Raman scattering (SERRS)," Chemical Physics Letters, vol. 352, no. 5-6, pp. 378-384, 2002.

[18] M. Brust, D. Bethell, C. J. Kiely, and D. J. Schiffrin, "Selfassembled gold nanoparticle thin films with nonmetallic optical and electronic properties," Langmuir, vol. 14, no. 19, pp. 5425-5429, 1998.

[19] D. Roy, J. Wang, and M. E. Welland, "Nanoscale imaging of carbon nanotubes using tip enhanced Raman spectroscopy in reflection mode," Faraday Discussions, vol. 132, pp. 215-225, 2006.

[20] M. I. Mishchenko, L. D. Travis, and A. A. Lacis, Scattering, Absorption, and Emission of Light by Small Particles, Cambridge University Press, Cambridge, UK, 2002.

[21] H. C. van de Hulst, Light Scattering by Small Particles, Dover, New York, NY, USA, 1981.

[22] I. Romero, J. Aizpurua, G. W. Bryant, and F. J. García de Abajo, "Plasmons in nearly touching metallic nanoparticles: singular response in the limit of touching dimers," Optics Express, vol. 14, no. 21, pp. 9988-9999, 2006.

[23] P. B. Johnson and R. W. Christy, "Optical constants of the noble metals," Physical Review B, vol. 6, no. 12, pp. 4370-4379, 1972.

[24] http://www.comsol.com/products/multiphysics/.

[25] M. Micic, N. Klymyshyn, and H. P. Lu, "Finite element method simulations of the near-field enhancement at the vicinity of fractal rough metallic surfaces," Journal of Physical Chemistry B, vol. 108, no. 9, pp. 2939-2947, 2004.

[26] J. P. Kottmann, O. J. F. Martin, D. R. Smith, and S. Schultz, "Spectral response of plasmon resonant nanoparticles with a non-regular shape," Optics Express, vol. 6, no. 11, pp. 213-219, 2000.

[27] J. Jin, The Finite Element Method in Electromagnetics, John Wiley \& Sons, New York, NY, USA, 2nd edition, 2002.

[28] A. Otto, "Surface-enhanced Raman scattering: "Classical" and "Chemical" origins," in Light Scattering in Solids IV, vol. 54 of Topics in Applied Physics, pp. 289-418, Springer, Berlin, Germany, 1984.

[29] F. J. García-Vidal and J. B. Pendry, "Collective theory for surface enhanced Raman scattering," Physical Review Letters, vol. 77, no. 6, pp. 1163-1166, 1996.

[30] K. Kneipp, Y. Wang, H. Kneipp, et al., "Single molecule detection using surface-enhanced Raman scattering (SERS)," Physical Review Letters, vol. 78, no. 9, pp. 1667-1670, 1997.

[31] H. Xu, J. Aizpurua, M. Käll, and P. Apell, "Electromagnetic contributions to single-molecule sensitivity in surfaceenhanced Raman scattering," Physical Review E, vol. 62, no. 3, pp. 4318-4324, 2000.

[32] Z.-Q. Tian and B. Ren, "Adsorption and reaction at electrochemical interfaces as probed by surface-enhanced Raman spectroscopy," Annual Review of Physical Chemistry, vol. 55, pp. 197-229, 2004.

[33] Z.-Q. Tian, B. Ren, and D.-Y. Wu, "Surface-enhanced Raman scattering: from noble to transition metals and from rough surfaces to ordered nanostructures," Journal of Physical Chemistry B, vol. 106, no. 37, pp. 9463-9483, 2002.
[34] L. A. Dick, A. D. McFarland, C. L. Haynes, and R. P. van Duyne, "Metal film over nanosphere (MFON) electrodes for surface-enhanced Raman spectroscopy (SERS): improvements in surface nanostructure stability and suppression of irreversible loss," Journal of Physical Chemistry B, vol. 106, no. 4, pp. 853-860, 2002.

[35] D. Jung, Y. M. Lee, Y. Lee, N. H. Kim, K. Kim, and J.-K. Lee, "Facile fabrication of large area nanostructures for efficient surface-enhanced Raman scattering," Journal of Materials Chemistry, vol. 16, no. 30, pp. 3145-3149, 2006.

[36] M. E. Abdelsalam, P. N. Bartlett, J. J. Baumberg, S. Cintra, T. A. Kelf, and A. E. Russell, "Electrochemical SERS at a structured gold surface," Electrochemistry Communications, vol. 7, no. 7, pp. 740-744, 2005.

[37] J. Lu, D. Chamberlin, D. A. Rider, M. Liu, I. Manners, and T. P. Russell, "Using a ferrocenylsilane-based block copolymer as a template to produce nanotextured Ag surfaces: uniformly enhanced surface enhanced Raman scattering active substrates," Nanotechnology, vol. 17, no. 23, pp. 5792-5797, 2006.

[38] N. M. B. Perney, J. J. Baumberg, M. E. Zoorob, M. D. B. Charlton, S. Mahnkopf, and C. M. Netti, "Tuning localized plasmons in nanostructured substrates for surface-enhanced Raman scattering," Optics Express, vol. 14, no. 2, pp. 847-857, 2006.

[39] R. M. Stockle, Y. D. Suh, V. Deckert, and R. Zenobi, "Nanoscale chemical analysis by tip-enhanced Raman spectroscopy," Chemical Physics Letters, vol. 318, no. 1, pp. 131-136, 2000. 

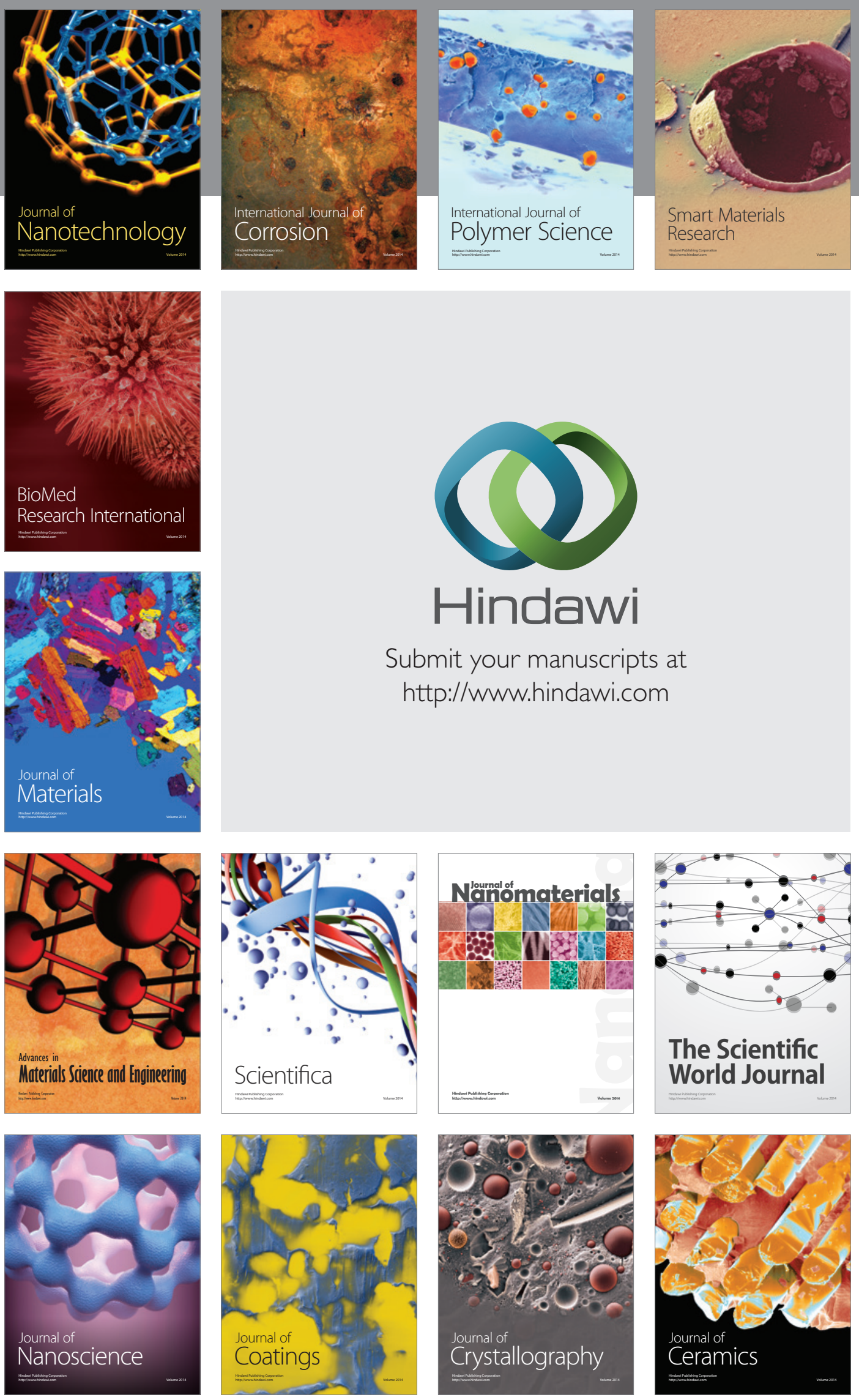

The Scientific World Journal

Submit your manuscripts at

http://www.hindawi.com

\section{World Journal}

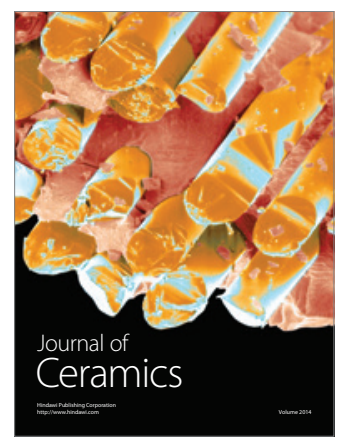

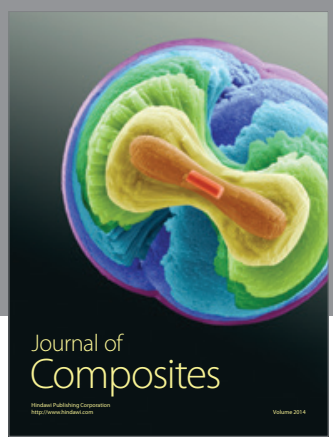
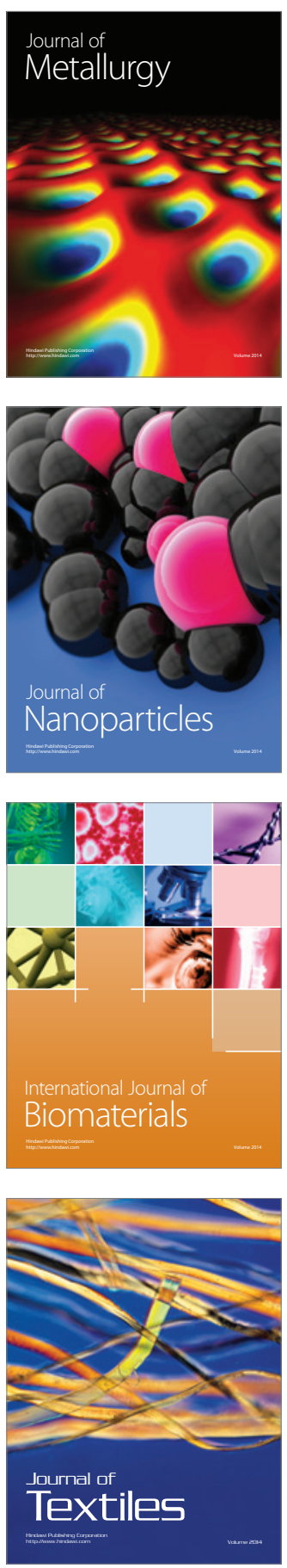